\title{
Numerical Simulation of Magnetohydrodynamic Forced Convective Boundary Layer Flow past a Stretching/Shrinking Sheet Prescribed with Variable Heat Flux in the Presence of Heat Source and Constant Suction
}

\author{
S. P. Anjali Devi ${ }^{1}$ and J. W. S. Raj ${ }^{2 \dagger}$ \\ ${ }^{1,2}$ Department of Applied Mathematics, Bharathiar University, Coimbatore, Tamil Nadu, India. \\ ${ }^{\dagger}$ Corresponding Author Email: wilfred_dphd@yahoo.com
}

(Received December 31, 2012; accepted April 3, 2013)

\begin{abstract}
A study has been carried out on MHD boundary layer forced convection flow along a shrinking surface with variable heat flux in the presence of heat source. The flow is generated due to linear shrinking of the sheet and is influenced by uniform transverse magnetic field. The basic boundary layer momentum and heat transfer equations, which are nonlinear partial differential equations, are converted into nonlinear ordinary differential equations by means of similarity transformation. Numerical solution of the resulting boundary value problem is obtained using Nachtsheim Swigert shooting iteration scheme for the satisfaction of asymptotic boundary conditions along with the Fourth Order Runge Kutta method. The effects of suction parameter, magnetic parameter, Prandtl number, heat source parameter, stretching/shrinking parameter and heat flux parameter on velocity and temperature are shown in several plots. The results are in good agreement with the earlier published works under some limiting cases. Skin friction coefficient and wall temperature are also explored for typical values of the parameter involved in the study.
\end{abstract}

Keywords: Boundary layer, Similarity solution, Variable surface heat flux temperature, Wall temperature, Stretching/shrinking sheet.

\section{NOMENCLATURE}

\author{
$B$ heat source parameter \\ $B_{0}$ magnetic field strength \\ $C_{f}$ skin friction coefficient \\ $C_{p}$ specific heat at constant pressure \\ $D$ positive constant \\ $F$ dimensionless velocity \\ $k$ thermal conductivity of the fluid \\ $M^{2}$ magnetic parameter \\ $n$ heat flux parameter \\ $\operatorname{Pr}$ Prandtl number

\section{Greek symbols} \\ $\theta$ dimensionless temperature \\ $\xi$ similarity variable \\ $\eta$ dimensionless coordinate \\ $\tau_{w}$ shear stress at the wall
}

\author{
$q_{w}$ heat flux at the surface \\ $Q$ internal heat generation \\ $S$ suction parameter \\ $T$ temperature of the fluid \\ $T_{w}$ temperature at the wall \\ $T_{\infty} \quad$ temperature of the free stream fluid \\ $u \quad$ velocity in the $x$ direction \\ $v$ velocity in the $y$ direction \\ $x$ dimensional distance along the sheet \\ $y$ dimensional distance normal to the sheet \\ $\varepsilon \quad$ stretching/shrinking parameter \\ $\rho$ density of the fluid \\ $\mu \quad$ dynamic viscosity of the fluid \\ $v$ kinematic viscosity of the fluid
}


$\sigma \quad$ electrical conductivity of the fluid

$\psi \quad$ stream function

\section{INTRODUCTION}

Boundary layer behaviour over a continuous moving solid surface is an important type of flow occurring in several engineering processes. The heat transfer due to a continuously stretching surface through an ambient fluid is one of the thrust areas of current research. Such investigations find their application over a broad spectrum of science and engineering process, especially in the field of chemical engineering. Many chemical engineering processes like metallurgical process, polymer extrusion involves cooling of a molten liquid being stretched into a cooling system.

Sakiadis (1961) initiated the study of the boundary layer flow over a continuous solid surface moving with constant speed. The boundary layer problem considered by Sakiadis differs from the classical boundary layer problem addressed by Blasius mainly due to the entrainment of the ambient fluid. Here the surface is assumed to be inextensible $\left(u_{w}=\right.$ 0 ) whereas most of the physical situations concern with extensible surfaces $\left(u_{w}=a x\right)$ moving in a cooling liquid. Crane (1970) was the first to consider the boundary layer behaviour over an extensible surface where the velocity of the surface varies linearly with the distance from the slit. The linear stretching problem for hydromagnetic case was studied by Chakrabarti and Gupta (1979). The effects of variable surface temperature and variable surface heat flux over the heat transfer characteristics of a continuous linear stretching surface was investigated by Chen and Char (1988).

Thermal boundary layer on a power law stretched surface with suction or injection was investigated by Ali (1995). Elbashbeshy (1998) examined the heat transfer over a stretching surface with variable surface heat flux. Liao (2005) obtained a new branch of solution of boundary layer flow over a permeable stretching plate. The micropolar transport phenomena over a stretching sheet were discussed by Bhargava et al. (2007). MHD flow of a micropolar fluid past a stretched permeable surface with heat generation or absorption was studied by Khedr et al. (2009). Dissipation effects on nonlinear MHD flow over a stretching surface with prescribed heat flux was examined by Anjali Devi and Ganga (2010). Radiative MHD flow over a non-isothermal stretching sheet in a porous medium was investigated by Paresh Vyas and Nupur Srivastava (2010). Azeem Shahzad et al. (2012) presented the exact solution for axisymmetric flow and heat transfer over a nonlinear radially stretching sheet.

The problem in the reverse case i.e., very little is known about the shrinking sheet where the velocity on the boundary is towards the origin. For this flow configuration, the sheet is shrunk towards a slot and the flow is quite different from the stretching out case. It is also shown that mass suction is required to maintain the flow over a shrinking sheet. Literature survey indicates that the flow induced by a shrinking Sheet recently gains attention of modern researchers for its interesting characteristics.

Shrinking sheet is a surface which decreases in size to a certain area due to an imposed suction or external heat. One of the most common applications of shrinking sheet problems in industries and engineering is shrinking film. In packaging of bulk products, shrink film is very useful as it can be unwrapped easily with adequate heat. Shrinking problem can also be applied to study the capillary effects in smaller pores, the shrink-swell behavior and the hydraulic properties of agricultural clay soils.

The existence and uniqueness of similarity solution of the equation for the flow due to a shrinking sheet with suction was established by Miklavcic and Wang (2006). MHD rotating flow of a viscous fluid over a shrinking surface was analyzed by Sajid et al. (2008). Closed form exact solution of MHD viscous flow over a shrinking sheet was examined by Fang and Zhang (2009) without considering the heat transfer. The application of homotopy analysis method for MHD viscous flow over a shrinking sheet was examined by Sajid and Hayat (2009). An analytical solution for thermal boundary layer flow over a shrinking sheet considering prescribed wall temperature and prescribed wall heat flux cases was investigated by Fang and Zhang (2010). Hayat et al. (2010) examined the analytical solution of shrinking flow of second grade fluid in a rotating frame. Ali et al. (2010) presented MHD flow and heat transfer due to a permeable shrinking sheet of a viscous electrically conducting fluid with prescribed surface heat flux. Noor et al. (2010) obtained simple non-perturbative solution for MHD viscous flow due to a shrinking sheet. The effect of heat source/sink on MHD flow and heat transfer over a shrinking sheet with heat transfer over a shrinking sheet with mass suction for constant surface temperature was analysed by Bhattacharyya (2011). Das (2012) investigated the effects of slip on MHD mixed convection stagnation flow of a micropolar fluid towards a shrinking vertical sheet.

So far no contribution has been made on steady, laminar, two dimensional boundary layer MHD flow of a viscous, incompressible electrically conducting fluid with heat transfer over a stretching/shrinking sheet prescribed with variable heat flux in the presence of magnetic field and uniform heat source. The stretching/shrinking sheet is subjected to suction. Nonlinear partial differential equations for momentum and energy are transformed into nonlinear ordinary differential equations by introducing suitable similarity transformations. Thus the obtained equations are solved numerically utilizing Nachtsheim Swigert shooting iteration scheme for the satisfaction of asymptotic boundary conditions together with the Fourth Order Runge Kutta method.

The effect of magnetic parameter, suction parameter, Prandtl number, heat source parameter and heat flux parameter over the flow field and 
other physical quantities are discussed with the help of numerical values by means of graphs. From the present results it is inferred that the presence of magnetic field and heat source alters the flow and heat transfer characteristics significantly.

\section{MATHEMATICAL ANALYSIS}

Consider a steady, two dimensional, laminar nonlinear hydromagnetic boundary layer flow of a viscous, incompressible, electrically conducting fluid, caused by a stretching/shrinking sheet subjected to suction in the presence of uniform transverse magnetic field. Cartesian coordinate system is chosen. The velocity components $u$ and $v$ are taken in $x$ and $y$ directions respectively. A magnetic field of strength $\boldsymbol{B}_{\mathbf{0}}$ is applied normal to the boundary.

The analysis is based on the following assumptions:

- The fluid has constant physical properties.

- The magnetic Reynolds number is assumed to be small so that the induced magnetic field is negligible.

- Since the induced magnetic field is assumed to be negligible and as $\boldsymbol{B}_{\mathbf{0}}$ is independent of time, curl $\boldsymbol{E}=0$. Also $\operatorname{div} \boldsymbol{E}=0$ in the absence of surface charge density. Hence $\boldsymbol{E}=0$ is assumed.

- The energy equation involves the heat source term and variable heat flux is prescribed at the stretching/shrinking surface.

- The effect of viscous and joules dissipation are assumed to be negligible in the energy equation.

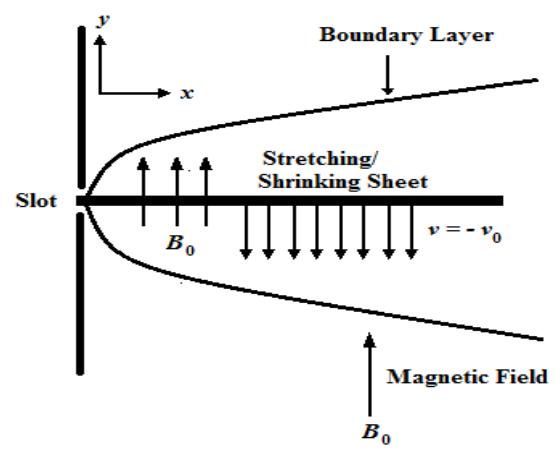

Fig. 1. Schematic diagram of the problem

Under these assumptions, the governing equations of the problem are given by:

$\frac{\partial u}{\partial x}+\frac{\partial v}{\partial y}=0$

$u \frac{\partial u}{\partial x}+v \frac{\partial u}{\partial y}=v \frac{\partial^{2} u}{\partial y^{2}}-\frac{\sigma B_{0}^{2}}{\rho} u$

$\rho C_{p}\left(u \frac{\partial T}{\partial x}+v \frac{\partial T}{\partial y}\right)=k \frac{\partial^{2} T}{\partial y^{2}}+Q\left(T-T_{\infty}\right)$

Boundary conditions pertaining to velocity are :

$u=b x, \quad v=-v_{0} \quad$ at $\quad y=0$ $u=0$

as $y \rightarrow \infty$

where $b<0$ is the shrinking constant and $b>0$ is the stretching constant.

\subsection{Flow Analysis}

Equations (1) and (2) admit a self similar solution. A stream function $\psi$ defined by :

$u=\frac{\partial \psi}{\partial y}, \quad v=-\frac{\partial \psi}{\partial x}$

is such that the continuity equation is identically satisfied. A dimensionless stream function $F(\eta)$ is given by :

$$
\psi(x, y)=\sqrt{a v} \quad x F(\eta), \eta=y \sqrt{\frac{a}{v}}
$$

where the similarity variable $\eta$ depends solely on $y$. Using Eqs. (6) and (7), the velocity components become :

$u=a x F^{\prime}(\eta), v=-\sqrt{a v} F(\eta)$

The wall mass transfer velocity becomes $v_{w}=-\sqrt{a v} F(0)$. Substituting for $u$ and $v$, Eq. (2) becomes:

$F^{\prime \prime \prime}+F F^{\prime \prime}-\left(F^{\prime}\right)^{2}-M^{2} F^{\prime}=0$

where the prime denotes derivative with respect to $\eta$ and $M^{2}=\frac{\sigma B_{0}^{2}}{\rho a}$ is the magnetic parameter. By using Eq. (8) in Eqs. (4) and (5), the boundary conditions of Eq. (9) become:

$F(0)=S, \quad F^{\prime}(0)=\varepsilon$ and $F^{\prime}(\infty)=0$

where $S=\frac{v_{0}}{\sqrt{a v}}\left(v_{0}>0\right)$ is the suction parameter and $\varepsilon=b / a$ is the stretching/shrinking parameter and $\varepsilon>0$ denotes the stretching sheet and $\varepsilon<0$ denotes shrinking sheet.

\subsection{Skin Friction Analysis}

For practical applications, the major physical quantity of our interest lies in the investigation of the flow behaviour by analyzing the nondimensional skin friction $C_{f}$ and its nondimensional parameters. The shear stress at the wall is given by :

$$
\tau_{w}=\mu\left(\frac{\partial u}{\partial y}\right)_{y=0}
$$

\subsection{Heat Transfer Analysis}

The thermal boundary conditions depend on the type of heating process under consideration. The case when the variable heat flux is prescribed on the stretching/shrinking surface is considered. The boundary conditions are:

$$
-k \frac{\partial T}{\partial y}=q_{w}=D x^{n} \quad \text { at } \quad y=0
$$

$T \rightarrow T_{\infty}$ as $y \rightarrow \infty$

The following similarity transformation is introduced to solve the energy equation.

$$
T-T_{\infty}=\frac{D x^{n}}{k} \sqrt{\frac{v}{a}} \theta(\eta)
$$

Substituting Eqs. (8) and (13), Eq. (3) takes the form

$\theta^{\prime \prime}+\operatorname{Pr} F \theta^{\prime}-n \operatorname{Pr} F^{\prime} \theta+\operatorname{Pr} B \theta=0$

where $\operatorname{Pr}=\frac{\mu C_{p}}{k} \quad$ and $B=\frac{Q}{a \rho C_{p}}$.

with its corresponding boundary conditions as: 
$\theta^{\prime}(0)=-1 \quad \theta(\infty)=0$

\section{NUMERICAL SIMULATION}

The effect of magnetic field on steady two dimensional flow of a viscous incompressible electrically conducting fluid past a shrinking sheet in the presence of heat source has been investigated numerically by using Nachtsheim Swigert iterative shooting technique along with Fourth Order Runge Kutta method. To integrate the momentum Eq. (9) and energy Eq. (14) the values of $F^{\prime \prime}(0)$ and $\theta(0)$ are required, but no such values are given in the boundary. Hence these equations which constitute boundary value problem are converted into an initial value problem using Nachtsheim Swigert shooting iterative technique. Based on convergence criterion, different initial guesses were made for different values of the pertinent parameters namely suction parameter, magnetic parameter, Prandtl number, heat source parameter, stretching/shrinking parameter and heat flux parameter. Once the suitable value for $F^{\prime \prime}(0)$ and $\theta(0)$ are chosen, the resulting initial value problem are then integrated using Fourth Order Runge Kutta method. The success of the procedure depends very much on how good the guess is. A systematic study on the effect of various parameters on flow and heat transfer characteristics is carried out.

\section{RESULTS AND DISCUSSION}

Effect of heat source on nonlinear MHD boundary layer flow and heat transfer over a stretching/shrinking surface with variable heat flux is presented. The numerical solution of the problem is obtained using Nachtsheim Swigert shooting iteration scheme for the satisfaction of asymptotic boundary conditions along with the Fourth Order Runge Kutta integration method.

Numerical values of the solution are obtained by fixing various values for the physical parameters involved in the problem namely suction parameter $S$, magnetic parameter $M^{2}$, Prandtl number $P r$, heat source parameter $B$, stretching/shrinking parameter $\varepsilon$ and heat flux parameter $n$. The effect of pertinent parameters on the velocity, temperature, skin friction coefficient and wall temperature are presented. The skin friction at the plate and the wall temperature for prescribed wall heat flux case are found.

In the absence of heat source and when constant heat flux is prescribed on the shrinking sheet the present results are identical to the results for the two dimensional case of Ali et al. (2011). This is evidently shown through Fig. 2.

Further it is seen that in the absence of heat source, the results for skin friction coefficient reduce to that of the results of Sajid and Hayat (2009) and Noor et al. (2010) for $m=1$. These are noted through Table 1 .

From the table it is seen that an increase in suction parameter, magnetic parameter, stretching/shrinking parameter and Prandtl number declines the wall temperature whereas it is increased by the heat flux parameter and heat source parameter.

Table 1 Comparison results of Skin Friction coefficient $F$ "'(0) when $S=1$ and $M^{2}=4$, $\varepsilon=-1$

\begin{tabular}{|c|c|c|}
\hline $\begin{array}{c}\text { Present } \\
\text { Study } \\
\text { (Author's) }\end{array}$ & $\begin{array}{c}\text { Sajid and } \\
\text { Hayat (2009) }\end{array}$ & $\begin{array}{c}\text { Noor } \text { et al. } \\
(2010)\end{array}$ \\
\hline 2.30278 & 2.30277 & 2.30278 \\
\hline
\end{tabular}

Table 2 depicts the effect of suction parameter, magnetic parameter, Prandtl number, stretching/shrinking parameter, heat source parameter and heat flux parameter on skin friction coefficient and wall temperature when the sheet is prescribed with variable wall heat flux.

Table 2 Values of Skin Friction Coefficient and Wall Temperature with $S=3, M^{2}=2$, $B=0.05, P r=0.71, \varepsilon=-1$ and $n=2$

\begin{tabular}{|c|c|c|}
\hline$F^{\prime \prime}(0)$ & $\theta(0)$ & $\begin{array}{c}\text { Physical } \\
\text { Parameters }\end{array}$ \\
\hline 2.414214 & 1.350447 & $S=2.0$ \\
\hline 3.302775 & 0.591394 & $S=3.0$ \\
\hline 4.236068 & 0.397867 & $S=4.0$ \\
\hline 5.192583 & 0.303990 & $S=5.0$ \\
\hline 6.162278 & 0.247320 & $S=6.0$ \\
\hline 2.618034 & 0.618505 & $M^{2}=0.0$ \\
\hline 3.000000 & 0.601904 & $M^{2}=1.0$ \\
\hline 3.302775 & 0.591394 & $M^{2}=2.0$ \\
\hline 3.561552 & 0.583764 & $M^{2}=3.0$ \\
\hline 3.791287 & 0.577819 & $M^{2}=4.0$ \\
\hline 3.302775 & 0.591394 & $P r=0.71$ \\
\hline 3.302775 & 0.405235 & $\operatorname{Pr}=1.00$ \\
\hline 3.302775 & 0.259253 & $\operatorname{Pr}=1.50$ \\
\hline 3.302775 & 0.162542 & $P r=2.30$ \\
\hline 3.302775 & 0.049805 & $\operatorname{Pr}=7.00$ \\
\hline 3.302775 & 0.584103 & $B=0.00$ \\
\hline 3.302775 & 0.591394 & $B=0.05$ \\
\hline 3.302775 & 0.595935 & $B=0.08$ \\
\hline 3.302775 & 0.599036 & $B=0.10$ \\
\hline 3.302775 & 0.633833 & $B=0.30$ \\
\hline 3.302775 & 0.445710 & $n=-2.0$ \\
\hline 3.302775 & 0.473477 & $n=-1.0$ \\
\hline 3.302775 & 0.506004 & $n=0.0$ \\
\hline 3.302775 & 0.544660 & $n=1.0$ \\
\hline 3.302775 & 0.591394 & $n=2.0$ \\
\hline 3.302775 & 0.591394 & $\varepsilon=-1.0$ \\
\hline 1.718246 & 0.522368 & $\varepsilon=-0.5$ \\
\hline-1.839725 & 0.436031 & $\varepsilon=0.5$ \\
\hline-3.791287 & 0.406670 & $\varepsilon=1.0$ \\
\hline
\end{tabular}

The effect of suction parameter over the nondimensional transverse velocity is shown through Fig. 3. The effect of porosity is to enhance 
the transverse velocity. The effect of suction parameter for each value of $S$ remains uniform as we move away from the wall. Figure 4 demonstrates the nondimensional longitudinal velocity for different values of $S$. It is observed that a steady increase in longitudinal velocity accompanies a raise in $S$ with all profiles tending asymptotically to the horizontal axis.

Figure 5 conveys the effect of suction parameter over skin friction coefficient against $M^{2}$. It is noted that the skin friction increases due to porosity in the plate. Figure 6 brings out the significance of suction parameter over the temperature distribution. It is noted that as suction parameter increases, the temperature of the flow field decreases. It is further noted that the thermal boundary layer thickness decreases due to increase in suction parameter.

The influence of magnetic field over the dimensionless transverse velocity $F(\eta)$ is elucidated in Fig. 7. The presence of magnetic field accelerates the transverse velocity of the flow field. It is quite interesting to note that, the effect of magnetic parameter remains uniform as we move away from the wall. The effect of magnetic field over the dimensionless longitudinal velocity is shown in Fig. 8 . The longitudinal velocity has the same effect as the suction parameter so as to accelerate the velocity.

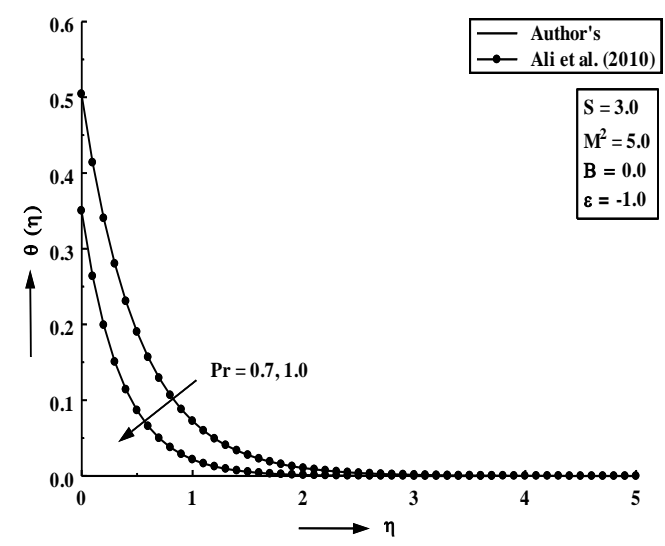

Fig. 2. Temperature profiles for various values of $\mathrm{Pr}$

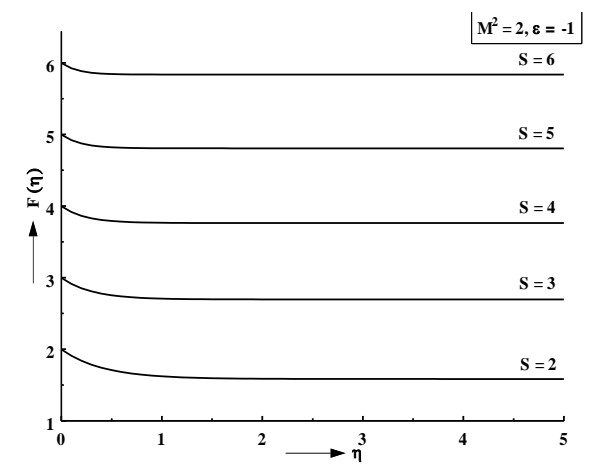

Fig. 3. Dimensionless transverse velocity profiles for various values of

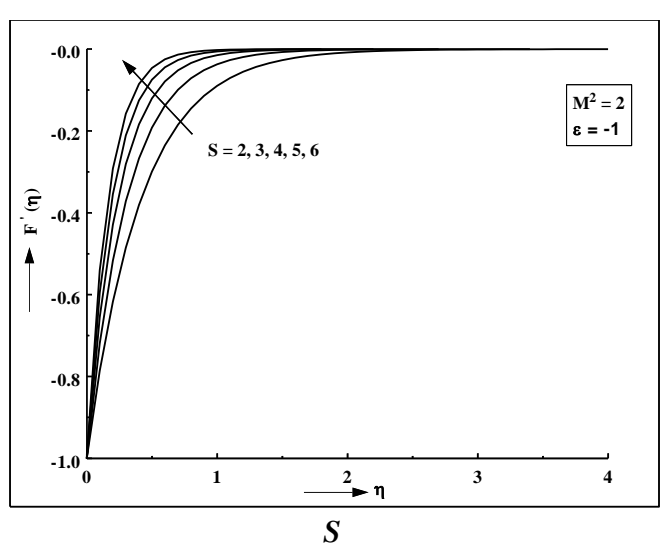

Fig. 4. Dimensionless longitudinal velocity profiles for various values of $S$

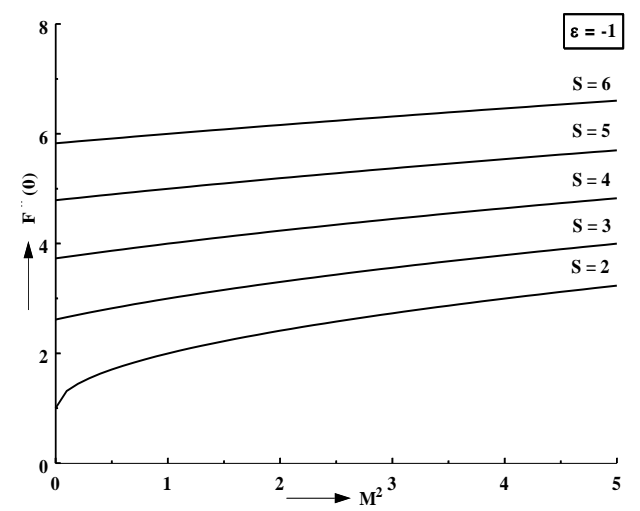

Fig. 5. Skin friction coefficient for various values of $S$

The increase in magnetic field strength decreases the temperature of the fluid and hence a reduction in thermal boundary layer thickness is demonstrated in Fig. 9.

Figure 10 presents the influence of Prandtl number over the dimensionless temperature distribution. It is seen that the effect of Prandtl number is to reduce the temperature.

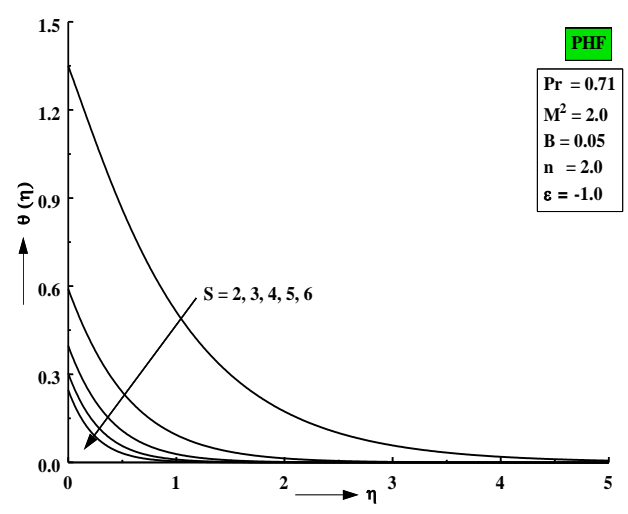

Fig. 6. Effect of $S$ over dimensionless temperature 


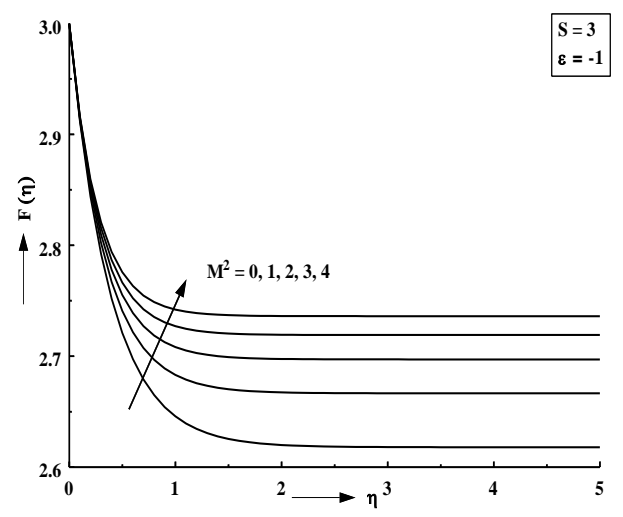

Fig. 7. Dimensionless transverse velocity profiles for various values of $\mathrm{M}^{2}$

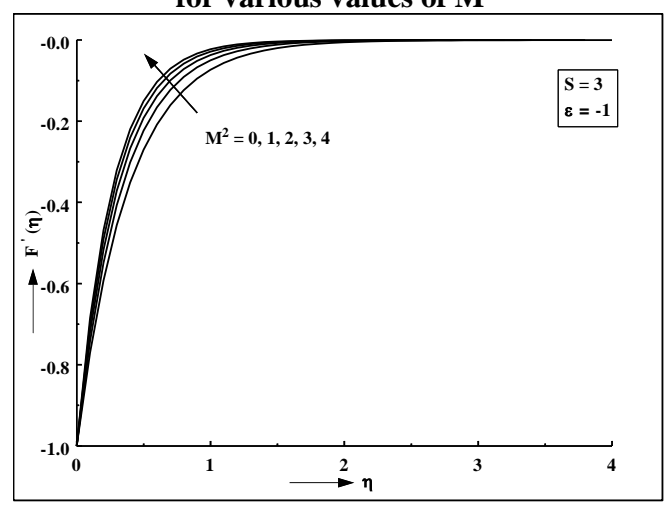

Fig. 8. Effect of $\mathrm{M}^{2}$ over dimensionless longitudinal velocity

The effect of heat source parameter on temperature distribution is shown in Fig. 11. It is observed that the heat source parameter increases the temperature of the fluid.

The graphical representation of the dimensionless temperature due to variation of heat flux parameter $n$ is seen in Fig. 12. As $n$ increases the temperature of the fluid also increases.

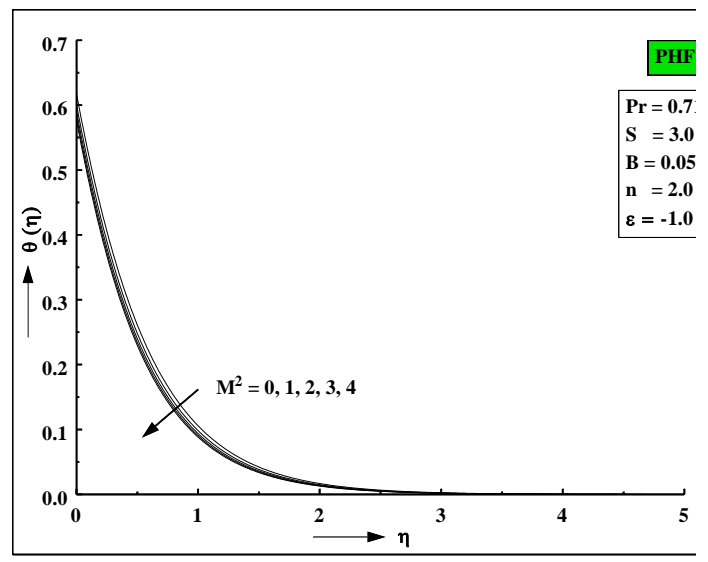

Fig. 9. Effect of $\mathrm{M}^{2}$ over dimensionless temperature

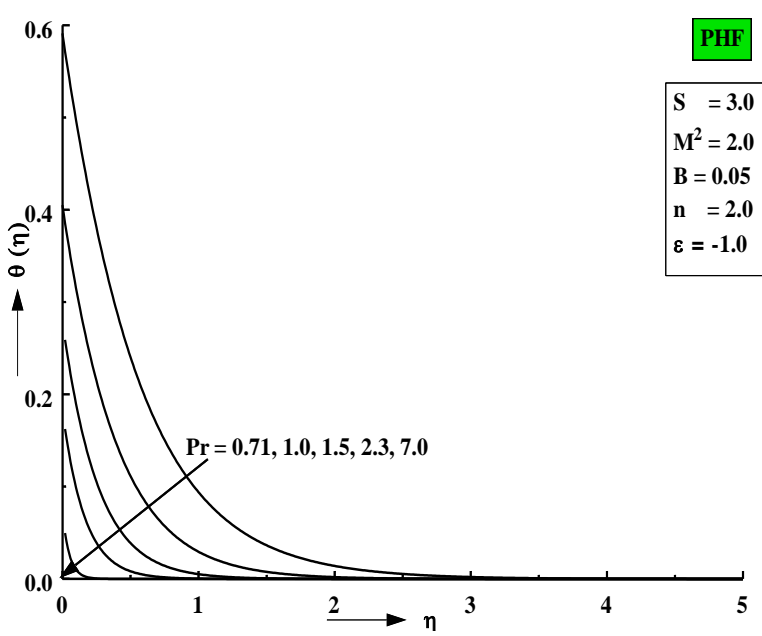

Fig. 10. Influence of Pr over dimensionless temperature

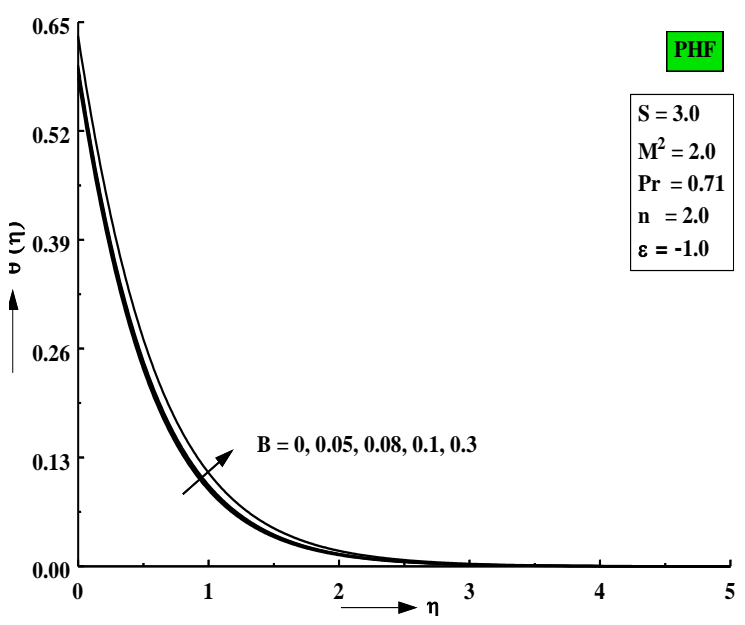

Fig. 11. Effect of $B$ over dimensionless temperature

Variation in longitudinal velocity for different stretching/shrinking parameter is elucidated in Fig. 13. The increasing effect of the parameter increases the longitudinal velocity significantly.

Figure 14 portrays the effect of stretching/shrinking parameter over the temperature distribution. The temperature of the fluid decreases due to its increasing effect. 


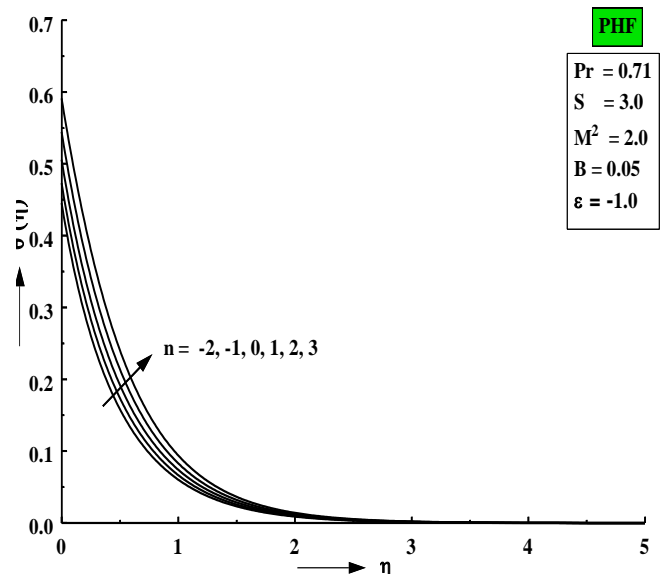

Fig. 12. Dimensionless temperature profiles for various $n$

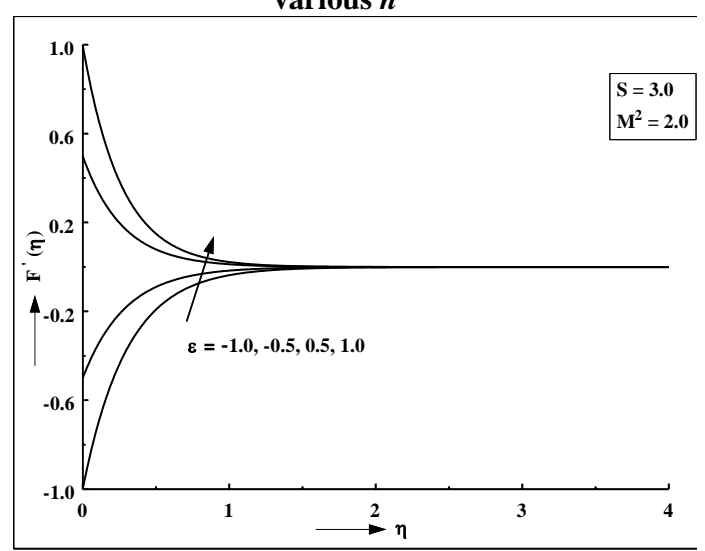

Fig. 13. Effect of stretching/shrinking parameter over longitudinal velocity

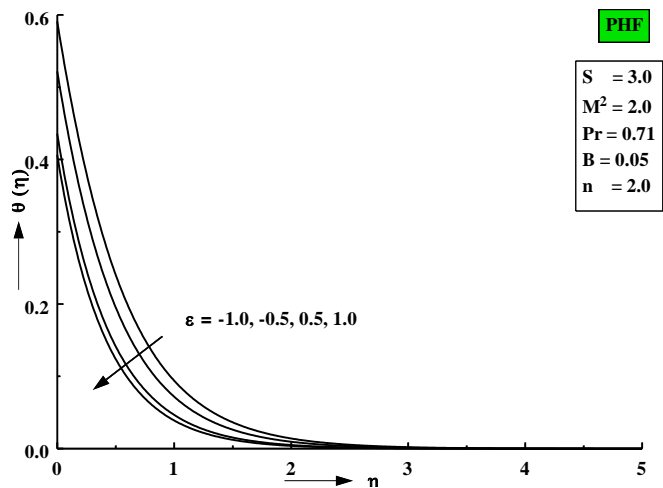

Fig. 14. Influence of stretching/shrinking parameter over dimensionless temperature

The effect of heat source is similar to that of Bhattacharyya (2011) so as to increase the temperature as heat source parameter increases.

eTemperature at the wall is found to be uniform in the results of Bhattacharyya (2011) while the temperature at the wall is nonuniform in this study. This is due to the reason that the variable heat flux temperature is prescribed on the shrinking sheet.

\section{CONCLUSION}

In general, the flow field and temperature distribution are affected by the physical parameters.

In the absence of magnetic field, heat source and when the fluid is electrically nonconducting $\left(M^{2}=\right.$ 0 ), the results are identical to that of Tiegang Fang and Ji Zhang (2010). Further in the absence of heat source our results are identical to that of Ali et al. (2010). In order to assure the accuracy of the applied numerical scheme the computed values of skin friction coefficient $F^{\prime \prime}(0)$ are compared with the available results of Sajid and Hayat (2009) and Noor et al. (2010) for two dimensional case and in the absence of heat source.

From the above results and discussion, the following conclusion are arrived

- The effect of suction parameter is to accelerate the transverse velocity and longitudinal velocity whereas the temperature of the flow field is decreased by the increasing value of suction parameter.

- Magnetic parameter increases both transverse and longitudinal velocity significantly. When the shrinking sheet is prescribed with variable surface heat flux temperature, the temperature of the fluid decreases with an increase in values of magnetic parameter.

- Suction parameter increases the skin friction with increase in magnetic parameter.

- The influence of Prandtl number is to decrease the temperature and hence the thermal boundary layer thickness reduces.

- The effect of heat source parameter is to increase the temperature of the fluid and thermal boundary layer thickness reduces with a decrease in heat source parameter.

- The heat flux parameter increases the temperature of the flow field significantly. It is noted that the thermal boundary layer thickness increases due to heat flux parameter $n$.

- The Suction parameter, magnetic parameter and Prandtl number decreases the wall temperature whereas it is increased by the heat source parameter and heat flux parameter $n$.

- It is believed that the results of the present work finds application in production engineering to upgrade the quality of the final product where the resulting heat transfer is very important.

\section{REFERENCES}

Sakiadis, B.C. (1961). Boundary layer behaviour on continuous solid surface: I boundary layer equations for two dimensional and axisymmetric flow. American Institute of Chemical Engineer J 7, 26-28.

Sakiadis, B.C. (1961). Boundary layer behaviour on continuous solid surface: II Boundary layer on 
S. P. Anjali Devi and J. W. S. Raj / JAFM, Vol. 7, No. 3, pp. 415-423, 2014.

a continuous flat surface. American Institute of Chemical Engineer J 7(2), 221-225.

Crane, L. J. (1970). Flow past a stretching plate. $Z$ Angew Math Physics 21(4), 645-647.

Chakrabarti, A and Gupta, A.S. (1979). Hydromagnetic flow and heat transfer over a stretching sheet. Quarterly of Applied Mathematics 37, 73-78.

Chen, C.K. and Char, M.I. (1988). Heat transfer of a continuously stretching surface with suction and blowing. Journal of Mathematical Analysis and Applications 135(2), 568-580.

Ali, M.E. (1995). On thermal boundary layer on a power-law stretched surface with suction or injection. International Journal of Heat and Fluid Flow 16(4), 280-290.

Elbashbeshy, E.M.A. (1998). Heat transfer over a stretching surface with variable surface heat flux. Journal of Physics D: Applied Physics 31(16), 1951-1954.

Liao, S.J. (2005). A new branch of solution of boundary layer flows over a permeable stretching plate. International Journal of Heat and Mass Transfer 48(12), 2529-2539.

Bhargava, R. Sharma, S. Takhar, H.S. and Bhargava, P (2007). Numerical solutions for Micropolar transport phenomena over nonlinear stretching sheet. Nonlinear Analysis: Modelling and Control 12(1), 45-63.

Khedr, M.E., Chamka, A.J. and Bayomi, M. (2009). MHD flow of a micropolar fluid past a stretched permeable surface with heat generation or absorption. Nonlinear Analysis: Modelling and Control 14(1), 27-40.

Anjali Devi, S.P. and Ganga, B. (2010). Dissipation effects on MHD nonlinear flow and heat transfer past a porous surface with prescribed heat flux. Journal of Applied Fluid Mechanics 3 (1), 1-6

Paresh Vyas and Nupur Srivastava (2010). Radiative MHD flow over a nonisothermal stretching sheet in a porous medium. Applied Math. Sci. 4(50), 2475-2484.
Azeem Shahzad, Ramzan Ali and Masood Khan (2012). On the exact solution for axisymmetric flow and heat transfer over a nonlinear radially stretching sheet. Chineese Physics Letter 29(8), 1-4

Miklavcic, M. and Wang, C.Y. (2006). Viscous flow due to a shrinking sheet. Quarterly of Applied Mathematics 64(2), 283-290.

Sajid, M. Javed, T. and Hayat, T. (2008). MHD rotating flow of a viscous fluid over a shrinking surface. Nonlinear dynamics 51(1), 259-265.

Fang, T. and Zhang, J. (2009). Closed-form exact solutions of MHD viscous flow over a shrinking sheet. Communication in Nonlinear Science and Numerical Simulation 14(7), 2853-2857.

Sajid, M. and Hayat, T. (2009). The application of homotopy analysis method for MHD viscous flow due to a shrinking sheet. Chaos Solitons and Fractals 39, 1317-1323.

Fang, T. and Zhang, J. (2010). Thermal boundary layers over a shrinking sheet: an analytical solution. Acta Mechanica 209(3-4), 325-343.

Hayat, T., Sania Iram, Javed, T. and Asghar, S (2010). Shrinking flow of second grade fluid in a rotating frame: An Analytic solution. Communications in Nonlinear Science and Numerical Simulation 15(10), 2932-2941.

Fadzilah MD Ali, Roslinda Nazar, Norihan MD Arifin. (2010). MHD viscous flow and heat transfer due to a permeable shrinking sheet with prescribed surface heat flux. WSEAS Transactions on Mathematics 5(9), 365-375. (ISSN: 1109-2769)

Noor, N.F.M. Awang Kechil, S. and Hashim, I. (2010). Simple non-perturbative solution for MHD viscous flow due to a shrinking sheet. Communications in Nonlinear Science and Numerical Simulation 15(2), 144-148.

Bhattacharyya, K. (2011). Effects of heat source/sink on MHD flow and heat transfer over a shrinking sheet with mass suction. Chemical Engineering Research Bulletin 15, 12-17. 
S. P. Anjali Devi and J. W. S. Raj / JAFM, Vol. 7, No. 3, pp. 415-423, 2014.

Das, K. (2012). Slip effects on MHD mixed convection stagnation point flow of a micropolar fluid towards a shrinking vertical sheet. Computers and Mathematics with Applications 63, 255-267. 